beautiful overview of his subject.

In the second group are articles of an astrophysical nature by Blandford and Rees. They deal with complicated phenomena that are only partially understood and not so well suited to systematic exposition. Faced with a difficult and wideranging task, both authors display not only the important facts and theories of high-energy astrophysics and cosmology, but also something of the special talents that are required to work successfully in these fields where all manner of different techniques - mathematical, statistical, physical, observational, speculative - are required to produce explanations of phenomena where many different physical effects intermingle to confuse us.

In the third group are articles of a more speculative variety on the frontiers of theory: Penrose describes the paradoxes of quantum reality and the possible role of gravity in the collapse of the wave function and the determination of the arrow of time; Linde, and Guth and Blau discuss the inflationary universe which the first two authors have done so much to develop and promote; Vilenkin manages to tell you all you need to know about cosmic strings in a 23-page article that is a model of clarity; Schwarz supplies a comprehensive yet readable contribution on superstrings and where he hopes they are going (only time will tell whether they get there via Stockholm); Hawking gives an update of his programme to calculate and interpret the wave function of the Universe, and explains his latest thinking about the arrow of time and the necessity for the weak anthropic principle to be employed in quantum cosmology; and Crnkovic and Witten provide a brief and somewhat technical exposition on canonical formalism. All the articles in this group deal with theoretical subjects that are as yet devoid of experimental test or decisive observational contact.

Finally, in the last group are an essay on newtonianism by Weinberg and the fascinating historical article by Israel. This charts the thinking and writings of astronomers on 'dark stars' from the eighteenth century to the present, and includes interesting accounts of his own thoughts at crucial stages in the interpretation of mathematical results about black hole mechanics and thermodynamics during the 1960 s and early 1970 s.

One of the strongest impressions to be gained from these articles is of the diversity of work in modern gravitation physics and the way in which gravitation is at last ceasing to be the aloof geometrical cousin of the rest of physics. In astrophysics it merges with the complexities of highenergy radiation processes to create spectacular physical phenomena. The search for gravitational waves exploits the latest technologies to provide astronomers with the promise of a new vista on the Universe. And in the inner space-time of elementary particles there are the first glimmerings of a convincing theory which might conjoin gravity with the other forces of Nature.

Who can doubt that Newton would be pleased but not surprised at these advances towards a unified description of the physical world and all its forces. For we find that 300 years ago, in the Preface to his stupendous book, he could write that, having determined "the motions of the planets, the comets, the moon and the sea", he was unfortunately unable to determine the remaining structure of the world from the same propositions because I suspect that they may all depend upon certain forces by which the particles of bodies, by some causes hitherto unknown, are either mutually impelled towards one another, and cohere in regular figures, or are repelled and recede from one another. These forces being unknown, philosophers have hitherto attempted the search of Nature in vain; but I hope the principles here laid down will afford some light either to this or some truer method of philosophy.

How right he was.

John D. Barrow is a Lecturer at the Astronomy Centre, University of Sussex, Brighton BNI $9 Q H, U K$.

\section{Skimming through space}

\section{James Trefil}

Space 2000: Meeting the Challenge of a New Era. By Harry L. Shipman. Plenum: 1987. Pp.431. \$19.95.

IN this book, University of Delaware astronomer Harry Shipman undertakes nothing less than a survey of space science and technology, both past and future, along with an introduction to the basic science (primarily astronomical) that is being done above the Earth's atmosphere. Despite the book's 400-plus pages, the sheer volume of the subject matter dictates that the coverage must of necessity be of the "broad and shallow" as opposed to the "deep and narrow" variety. Thus, the book is not intended for those already familiar with the space programme, but as an introduction for those who are not.

The book is divided into three sections. The first concerns the basic physics of satellite motion, the history of the space programme and an analysis of space technology. As you might expect from an author who has been deeply involved in the programme and was, in addition, the public relations and education officer of the American Astronomical Society, this part is very well done. Shipman's extensive experience with the space programme comes through very clearly, and there are many useful insights. To cite one among many, he points out a fundamental difference between the proposed Space Station and the Shuttle - an obvious distinction often overlooked by the Station's critics: the Space Station is modular, which means that unlike the Shuttle it can be built in stages; building it will not, therefore, necessarily drain money from other programmes, as the all-or-nothing Shuttle programme did in the 1970 s. I
Gone but not forgotten - the dodo head preserved in the Ashmolean Museum, Oxford, as depicted in H.E. Strickland and A.G. Melville's The Dodo and Its Kindred of 1848; the rest of the specimen (the right foot apart) was thrown out in 1755, by which time it could not be replaced. The picture is reproduced from Extinct Birds by Errol Fuller, published today in Britain by Viking/Rainbird, price $£ 20$. In the United States the book will be published by Facts on File.

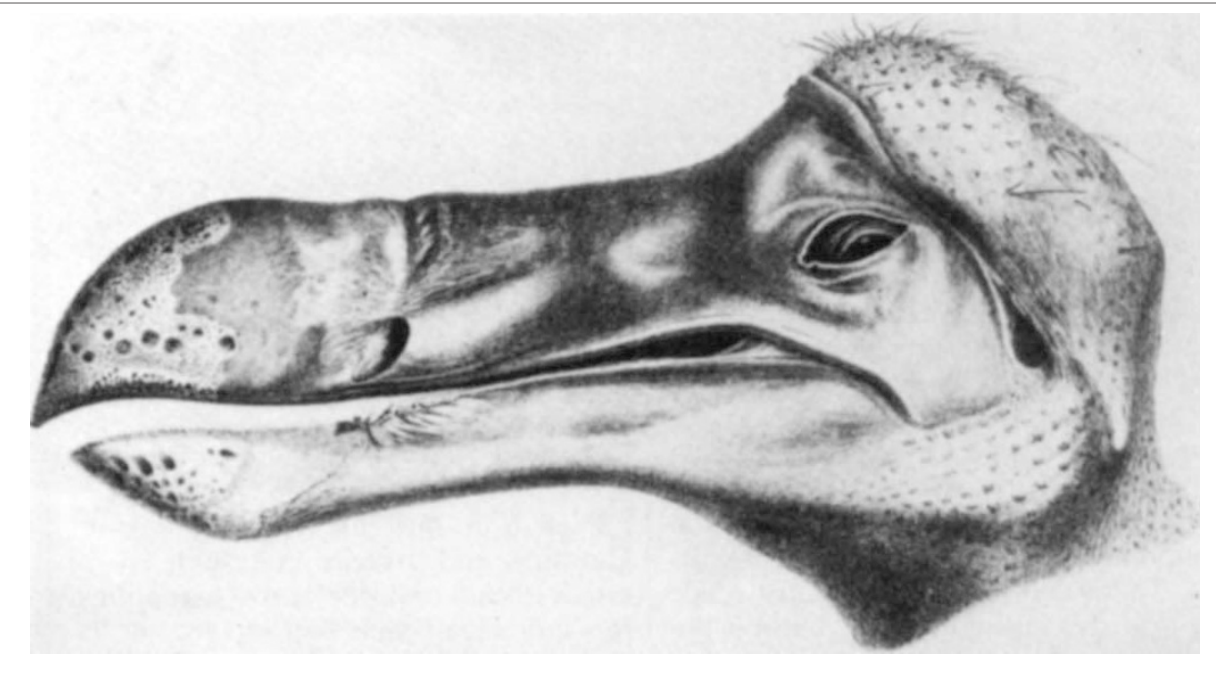

